\title{
Gene introgression in assessing fitness costs associated with phosphine resistance in the rusty grain beetle
}

\author{
Virgine T. Singarayan ${ }^{1} \cdot$ Rajeswaran Jagadeesan $^{2} \cdot$ Manoj K. Nayak $^{2} \cdot$ Paul R. Ebert $^{1} \cdot$ Gregory J. Daglish $^{2}$
}

Received: 28 June 2020 / Revised: 30 November 2020 / Accepted: 4 December 2020

(c) Crown 2021

\begin{abstract}
The current study investigates the fitness cost associated with phosphine resistance in the rusty grain beetle, Cryptolestes ferrugineus (Stephens), a problematic pest in the stored commodities that has developed strong resistance to fumigant phosphine. Three characterised insect strains: the susceptible (Ref-S), the strongly resistant (Ref-R), the introgressed resistant (Intro-R) and a segregating population $\left(F_{25}\right)$ derived from crossing the Ref-S and Ref-R strains were used in this study. Intro-R was developed by introgressing two phosphine resistance genes, cf_rph1 and cf_rph2 into Ref-S, aimed to reduce the influence of background genetic factors. Intro-R exhibited $592 \times$ resistance to phosphine and homozygous for strong resistance allele, cf_rph2 (L73N). Two key fitness cost criteria, developmental time and fecundity, were assessed under optimal and suboptimal conditions (less favourable diet and low temperature). There was no significant difference in developmental time and fecundity between Ref-S and either Intro-R strain or $F_{25}$ under optimal conditions. When challenged with a less favourable diet, cracked wheat + cracked sorghum $(\mathrm{CW}+\mathrm{CS})$, or exposed to a low temperature $\left(22^{\circ} \mathrm{C}\right)$, both Intro-R and Ref-S had similar developmental time and total numbers of $F_{1}$ progeny, confirming the absence of significant fitness effects expressed in these conditions. Therefore, we conclude that strongly phosphine resistant $C$. ferrugineus are unlikely to incur potential fitness costs. This finding will have implications towards developing strategies to manage this pest.
\end{abstract}

Keywords Biological cost $\cdot$ Developmental time $\cdot$ Reproduction $\cdot$ Suboptimal condition $\cdot$ Low temperature

\section{Key message}

- Cryptolestes ferrugineus has developed high levels of resistance to fumigant insecticide phosphine, posing threat to global grain trade

- A near isogenic phosphine resistant strain of C. ferrugineus was established to investigate potential fitness costs associated with two phosphine resistance genes

Communicated by Emmanouil Roditakis .

Virgine T. Singarayan

v.singarayan@uq.edu.au

1 School of Biological Sciences, The University of Queensland, St Lucia, Brisbane QLD 4072, Australia

2 Department of Agriculture and Fisheries, EcoSciences Precinct, GPO Box 267, Brisbane, Queensland QLD 4001, Australia
- Strongly phosphine resistant $C$. ferrugineus are unlikely to incur significant fitness costs, even when the insects were challenged with suboptimal conditions

- This finding provides a basis for developing phosphine resistance management strategies to manage $C$. ferrugineus

\section{Introduction}

Across the globe, fumigant phosphine is considered as a preferred treatment to disinfest stored commodities, due mostly to its cheap price, versatility in application and acceptance as a residue-free treatment by markets. Exclusive use of this valuable fumigant over the years, however, is threatening its future sustainability, with the development of heritable resistance to phosphine in major pest species (Benhalima et al. 2004; Lorini et al. 2007; Nayak et al. 2013, 2020; Daglish et al. 2014; Kaur et al. 2015; Kocak et al. 2015; Nguyen et al. 2016; Konemann et al. 2017; Afful et al. 2018; Agrafioti et al. 2019). Apart from being widespread over the 
last two decades, there has been significant increase in both the strength and frequency of phosphine resistance in several insect species (Holloway et al. 2016; Collins et al. 2017; Nayak et al. 2017) including the rusty grain beetle, Cryptolestes ferrugineus (Stephens) (Nayak et al. 2013). The level of resistance to phosphine reported in this pest species based on $\mathrm{LC}_{50}$ (lethal concentration to kill $50 \%$ of the population) is $1450 \times$ compared to its susceptible counterparts, the highest level ever reported among any of the insect species so far in Australia (Nayak et al. 2013). Strongly resistant C. ferrugineus can be controlled with either higher phosphine concentrations over longer exposure periods than that has been recommended for other species (Kaur and Nayak 2015), or using an alternative fumigant sulfuryl fluoride (Jagadeesan et al. 2017). To be successful, however, fumigation with these fumigants requires optimum levels of gas-tightness, which can be difficult to achieve in many existing storage structures.

Fitness costs are basically the consequences in terms of growth and development of an insect for being resistant to an insecticide or fumigant, and this can alter the rate and spread of resistance development (McKenzie 1996). Therefore, determining any such biological vulnerability associated with phosphine resistance in the absence or infrequent use of phosphine in C. ferrugineus will have important implications towards the designing and implementation of appropriate management practices against this key pest in the field.

Although several methods have been used to measure the fitness costs, a simple and common approach is the comparison of biological traits among the susceptible and resistant insect strains, referred as the 'between-population approach' (Alyokhin and Ferro 1999; Oliveira et al. 2007; Gassmann et al. 2009). A drawback in this approach, however, is that it fails to account for genetic variation among the strains and can confound the results (Raymond et al. 2005). To overcome this, some researchers have used 'population cage approach', in which resulting filial generations from hybridising resistant and susceptible strains in the absence of insecticide exposure are screened for frequency of resistant phenotypes/genotypes (Schlipalius et al. 2008; Daglish et al. 2014, 2015). No change in frequency in segregating populations after $F_{2}$ would indicate that the resistance is not associated with fitness cost. This population cage approach provides only certain degree of control for the variation in genetic background, which could otherwise modify the results of fitness assay, in particular if there is any epistatic gene interactions involved (Zhu et al. 2016).

Taking these shortcomings into account, the most accurate way to assess fitness costs associated with a resistant trait would be generating near-isogenic strains following a gene introgression approach, in which the susceptible and resistant strains being compared share a similar genetic background, except for the resistance genes (Roush and
McKenzie 1987). This allows the pleiotropic effects of the resistance gene to be isolated and provides an extra assurance that the variation in fitness cost is purely by the variants (mutations) within the resistance gene itself. The gene introgression approach had been used to study the fitness cost associated with malathion resistance in $C$. ferrugineus (White and Bell 1990) and highlighted the advantage of this approach over simply comparing the susceptible and resistant populations.

Fitness costs associated with phosphine resistance in stored product insects have been well researched and involved different approaches. Several studies established fitness cost associated with phosphine resistance in major pest species by comparing their biological parameters, such as reproduction and developmental time, particularly in the resistant and susceptible populations of the red flour beetle Tribolium castaneum (Herbst), the lesser grain borer, Rhyzopertha dominica (F.), and the saw-toothed grain beetle, Oryzaephilus surinamensis (L.) (Pimentel et al. 2007; Sousa et al. 2009; Bajracharya et al. 2016). In these studies, the researchers looked for significant relationships between the biological parameters and the resistance factor of the population. The genetic composition of the unselected field samples used in these studies was unknown but could have contained multiple genotypes. While laboratory selection may not reflect closely selection that occurs in the field, it is fundamental to genetic studies and allows deep investigation of individuals with known genetic characteristics.

Two studies compared the mobility of strains of stored grain insects and found no significant difference in walking and resource localisation abilities between susceptible and resistant strains of $R$. dominica and T. castaneum (Kaur et al. 2013; Sakka et al. 2020). In these studies, however, the researchers did not control for genetic background. Malekpour et al. (2018) demonstrated that phosphine resistance genes, introgressed into susceptible insects, had pleiotropic effects on movement behaviour of T. castaneum with slower resource location and flight behaviour in the resistant insects. These differing conclusions on mobility parameters can be regarded as the result of difference in methodologies used.

Daglish et al. $(2014,2015)$ used the population cage approach and established that there have been no fitness costs associated with phosphine resistance in weakly resistant $T$. castaneum and the rice weevil, Sitophilus oryzae (L.). The strong resistance to phosphine is conferred by synergistic interaction of two genes rph1, cytochrome b5 fatty acid desaturase (Cyt-b5-r) (Schlipalius et al. 2018) and $\mathrm{rph} 2$, dihydrolipoamide dehydrogenase (dld) (Schlipalius et al. 2012). The disruption of Cyt-b5-r in resistant insects decreases the polyunsaturated fatty acid content in membranes, while the mutation in dld disrupts generation of reactive oxygen species (ROS); both limit the potential for lipid peroxidation, a well-known toxic response induced 
by phosphine (Chaudhry and Price 1992) in insects. This synergistic interaction of $\mathrm{rph} 1$ and $\mathrm{rph} 2$, conferring strong resistance in $R$. dominica (Schlipalius et al. 2002), allowed Schlipalius et al. (2008) to monitor the relative fitness of rd_rph2 allele over 20 generations. The authors found no change in frequency of resistance alleles. This reinforces the general view that resistance to phosphine is not associated with fitness cost. On the contrary, Jagadeesan et al. (2013a) and Nguyen et al. (2016) have observed a significant reduction in frequency of resistant alleles over 18-1 generations and reported the possible association of fitness disadvantages in T. castaneum and S. oryzae. These differences in results among the species suggest that the fitness outcome is either species specific or depends on specific resistant alleles (mutations) and their genetic interactions. Furthermore, the above-mentioned studies had no complete control for inherent genetic variations. Recently, Daglish et al. (2020) utilised the gene introgression approach aiming to achieve complete control for genetic background by establishing an isogenic resistant $T$. castaneum and found no evidence for fitness costs for being strongly resistant to phosphine.

In $C$. ferrugineus, the subject of fitness in phosphine resistant individuals is yet to be investigated, despite its emerging pest status with the detection of highest levels of resistance as well as discovery of additional genetic factors not seen in other species (Jagadeesan et al. 2016). Thus, the current study was designed with two aims: 1 . to investigate the fitness cost (if any) in strongly resistant $C$. ferrugineus in an ideal situation with a complete control for genetic background, where the measured cost is exclusively attributed to phosphine resistance genes and 2. to quantify such costs (if any) in $F_{25}$ segregating population to reflect a field population with multiple genotypes. To achieve this, we established an introgressed resistant $C$. ferrugineus strain, which has similar genetic background as susceptible strain and a segregating population $\left(F_{25}\right)$ of the cross between the phosphine susceptible and resistant strains. These strains and $F_{25}$ were also challenged with suboptimal conditions (less favourable diet and low temperature) with an assumption that magnitude of fitness effects is more evident under challenging conditions (Raymond et al. 2005).

\section{Materials and methods}

\section{Reference insect strains}

We used a reference phosphine susceptible strain, Ref-S (QCF31), and a reference strongly resistant strain, Ref-R (QCF73), hereafter referred to as Ref-S and Ref-R, respectively. Both strains were originally derived from field samples collected in Australia: Ref-S collected in 1988 from Cecil Plains in Queensland, and Ref-R collected in 2007 from Edgeroi in New South Wales (Nayak et al. 2013). Both the strains are maintained in a dietary medium of rolled oats at a favourable temperature of $30^{\circ} \mathrm{C}$ for several generations. The Ref-R strain was confirmed to be strongly resistant after collection from the field and was exposed to phosphine selections (see phosphine fumigation and bioassay for details) once in a year $(1.0 \mathrm{mg} / \mathrm{L}$ for $168 \mathrm{~h})$, to ensure the maintenance of homozygosity of the trait (Nayak et al. 2013; Jagadeesan et al. 2016). Ref-R has been previously reported to be $1341 \times$ more resistant than the Ref-S based on the $\mathrm{LC}_{50}$ value for adults fumigated for $48 \mathrm{~h}$ (Nayak et al. 2013).

\section{Introgressed phosphine resistant strain}

Phosphine resistance genes from Ref-R strain were introgressed into the Ref-S by a step-wise process involving repeated backcrossing, sib-mating and selection for phosphine resistance genes as diagrammatically presented in Fig. 1, to generate the introgressed resistant strain (Intro-R). Original and reciprocal crosses were established between the Ref-R and Ref-S, and the resulting $F_{3}$ populations were pooled. Insects from this $F_{3}$ generation were subjected to discriminating selection with phosphine $(0.1 \mathrm{mg} / \mathrm{L}$ for $72 \mathrm{~h})$ that would exhibit $75 \%$ mortality in a segregating population to isolate homozygous resistant individuals (Jagadeesan et al. 2016). Approximately, 100 male survivors were identified and used to initiate the process of introgression. These resistant males were backcrossed with an equivalent number of virgin Ref-S females, and the resulting backcross progeny $\left(\mathrm{BC}_{1}-F_{1}\right)$ was allowed to mate randomly (sib-mating) to produce the $\mathrm{BC}_{1}-F_{2}$. Adult beetles of the $\mathrm{BC}_{1}-F_{2}$ were selected $(0.1 \mathrm{mg} / \mathrm{L}$ for $72 \mathrm{~h})$, and the surviving $\mathrm{BC}_{1}-F_{2}$ males were backcrossed with virgin Ref-S females, and this cycle of crossing followed by discriminatory selection was repeated until the $\mathrm{BC}_{3}-F_{2}$. At this stage, the Intro-R was expected to have $93.8 \%$ genetic identity with the Ref-S except for the two phosphine resistance genes, cf_rph1 and cf_rph2. Before starting the fitness experiments, the Intro- $R$ was also subjected to sequential selections with increasing phosphine concentration of $0.1,0.4,2.0$ and $4.0 \mathrm{mg} / \mathrm{L}$ over $72 \mathrm{~h}$ exposure period, respectively, over four subsequent generations to achieve homogeneity within the test cohort that would provide linear mortality responses in bioassays.

\section{Segregating population $\left(F_{25}\right)$ from the Ref-R $\times$ Ref-S cross}

A segregating population resulting from a single pair cross between Ref-R and Ref-S was maintained for 25 generations in the absence of phosphine selection (Jagadeesan et al. 2016). Phosphine resistance was characterised phenotypically in the $F_{24}$ generation (see Phosphine fumigation and bioassay), and fitness assays were undertaken on the $F_{25}$ 


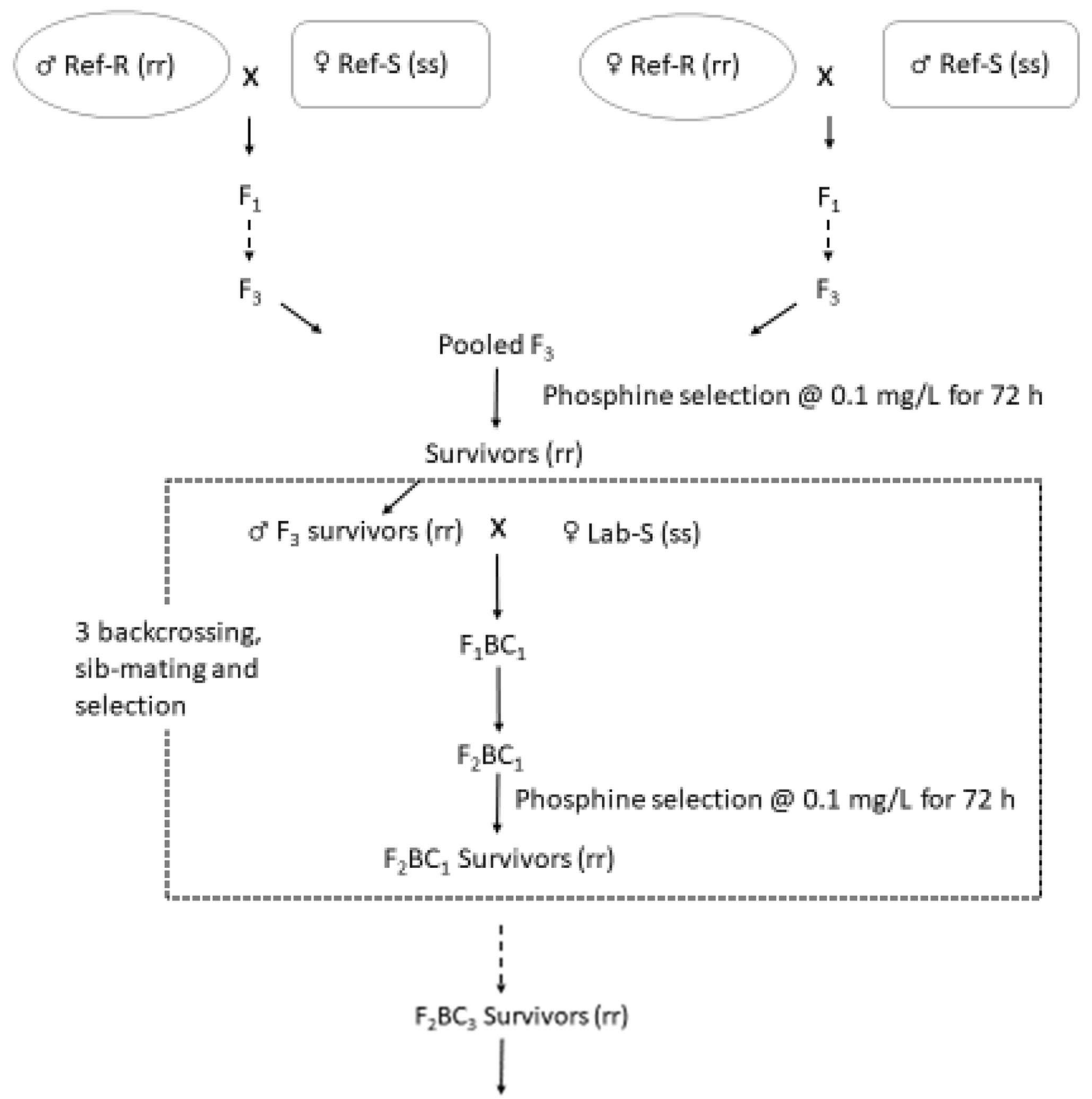

Serial selection with phosphine $0.1,0.4,2.0$ and $4.0 \mathrm{mg} / \mathrm{L}$ for $72 \mathrm{~h}$

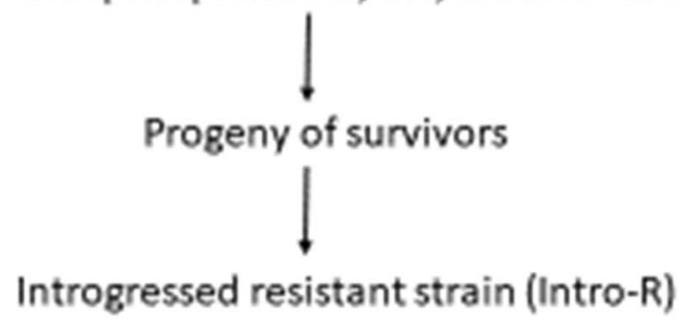

Fig. 1 Construction of introgressed resistant strain (Intro-R) from a cross between reference resistant (Ref-R) and reference susceptible strain (Ref-S) in Cryptolestes ferrugineus 
generation. A delay of one generation between the characterising of resistance and the fitness testing was necessary in order to obtain sufficient insect populations for both experiments. With two major genes conferring resistance in $C$. ferrugineus (Jagadeesan et al. 2016), $F_{2}$ and further filial generations of the cross between resistant and susceptible strain will contain nine genotypes ranging from fully susceptible (s1s1: s2s2) to fully strongly resistant (r1r1: r2r2). Therefore, the $F_{24}$ or $F_{25}$ generations could have contained up to nine insect genotypes. This segregating population represented a typical field population containing multiple genotypes.

\section{Phosphine fumigation and bioassay}

Phosphine gas was generated into a collection tube from commercial aluminium phosphide tablets $(56 \%)$ that were immersed in an aqueous solution of sulphuric acid (5\%). This source gas concentration was measured on a Clarus 580 gas chromatograph (PerkinElmer, Waltham, MA) using a thermal conductivity detector with nitrogen as the standard. The measured source concentration $\left(N_{1}\right)$ of phosphine $(\mathrm{mg} / \mathrm{L})$ and volume of desiccators $\left(V_{1}\right)$ were used to estimate the required volume $\left(V_{2}\right)$ of phosphine gas $(\mu L)$ to achieve the desired concentration $\left(\mathrm{N}_{2}\right)$ using the standard equation $V_{1} N_{1}=V_{2} N_{2}$ (Jagadeesan and Nayak 2017). The fumigation was performed by drawing the required volume of the gas from the collection tube using Hamilton air-tight gas syringe and injected into the desiccator chamber through the rubber septum.

Dose-mortality response bioassays were performed on the Ref-S, Ref-R, Intro-R and the $F_{24}$ segregating population to determine their strength of resistance to phosphine. Four concentration ranges were used: $0.002-0.05,1.0-8.0$, 0.5-6.0 and 0.005-3.0 mg/L for the strains Ref-S, Ref-R, Intro-R and $F_{24}$; respectively. Approximately, 200-400 adult beetles of 1-2 week post-emergence were fumigated for each concentration in gas tight desiccators at $20^{\circ} \mathrm{C}$ and $60 \% \mathrm{RH}$. The fumigation lasted for $72 \mathrm{~h}$ and mortality was assessed 7 days after the termination of fumigation (Jagadeesan et al. 2016). The mortality response data were analysed using probit analysis with concentration/log-concentration as explanatory variable (GenStat Version 11, 2008), and key parameters $\left(\mathrm{LC}_{50}\right.$ and $\left.\mathrm{LC}_{99.9}\right)$ were estimated.

\section{Gene sequencing to confirm introgression of cf_ rph2}

Ideally, introgression of both the genes, rph1 (cytochrome b5 fatty acid desaturase) and rph2 (dihydrolipoamide dehydrogenase) should have been confirmed in the Intro-R through molecular screening for cf_rph1 and cf_rph2. However, the mutation in the cf_rph1 in Ref-R of C.ferrugineus has a sequence deletion that makes amplification and sequencing of cf_rph1 through genomic DNA, a difficult task. Therefore, introgression of cf_rph1 was confirmed through phenotype assays alone (see Results: Phosphine bioassays). As insects need to be homozygous for rph1 and rph2 to be fully strongly resistant, confirmation that Intro- $\mathrm{R}$ is strongly resistant through phenotype assays would also confirm introgression of rph1.

In the case of cf_rph2, the mutation is target site single nucleotide polymorphism thus specific fragment of the cf_rph2 gene containing the functional mutation was amplified from genomic DNA and sequenced for polymorphisms. Genomic DNA was extracted from four individual insects using a modified Hotshot DNA extraction method described by (Montero-Pau and Gomez, 2008). A fragment of the $C$. ferrugineus dld gene that encompasses a genetic variant, L73N (Jagadeesan et al. 2020), linked with phosphine resistance was amplified using the primers cf_rph2_fwd (TGT GTCCTCTTCACGTATGT) cf_rph2_rev (TCATGCCCA ATTGGGCTGC). The PCR reaction mixture contained TERRA (Clontech) PCR $2 X$ buffer $25.0 \mu \mathrm{L}$, forward primer $(10 \mathrm{mM}) 2.0 \mu \mathrm{L}$, reverse primer $(10 \mathrm{mM}) 2.0 \mu \mathrm{L}$, water 16.0 $\mu \mathrm{L}$, Taq polymerase $1.0 \mu \mathrm{L}$, and $4 \mu \mathrm{L}$ of DNA template. Temperature cycling conditions were as follows: $95^{\circ} \mathrm{C}$ for $5 \mathrm{~min}$, followed by 40 cycles of $95^{\circ} \mathrm{C}$ for $30 \mathrm{~s}, 57^{\circ} \mathrm{C}$ for $30 \mathrm{~s}$, and $72{ }^{\circ} \mathrm{C}$ for $60 \mathrm{~s}$, with a final extension at $72{ }^{\circ} \mathrm{C}$ for 5 min, after which the temperature was held at $4{ }^{\circ} \mathrm{C}$ for $15 \mathrm{~min}$. Resulting amplicons were pooled and submitted to the Australian Genomic Research Facility (AGRF, www. agrf.com.au) for Sanger sequencing.

\section{Fitness cost experiments}

In the first set of experiments, developmental time of each representative experimental strain was assessed on a previously established favourable diet for this species (Jagadeesan et al. 2013b) at an optimal (30 $\left.{ }^{\circ} \mathrm{C}\right)$ (Rillett 1949; Ashby 1961) and a suboptimal low $\left(22^{\circ} \mathrm{C}\right)$ temperature (Fields 1992) using controlled environment rooms with regime of $70 \%$ RH. For each strain, 100 unsexed adults (1-2 weeks post-emergence) were released into a glass jar of $500 \mathrm{~mL}$ volume, filled with $100 \mathrm{~g}$ of rolled oats plus yeast $(20: 1)$ and sealed with perforated lid containing Whatman filter paper. We considered 100 adults in $100 \mathrm{~g}$ of medium to be low density based on the results of White et al. (1995) in which the mortality was low at densities as high as 1000 adults in $100 \mathrm{~g}$ of wheat or maize. The parental adults were allowed to lay eggs for 7 days and cleared thereafter. The glass jars with immatures were monitored regularly for adult emergence, starting from 14 days after the removal of parentals. The numbers of adult progeny emerged were recorded at 1-3-day intervals and sieved out until no more progeny had emerged.

The reproduction rate in each strain was measured similarly to the method described for developmental time in a 
different set of jars set up in parallel, but the numbers of adult progeny that emerged $\left(F_{1}\right)$ in each strain was recorded at one time along with their cohort weight, 39-71 days after the removal of parental adults, depending on the experimental conditions. To avoid repeated disturbance on developing progenies, a single cut-off time to record total emergence was chosen based the results of the developmental time experiment.

In the second set of experiments, developmental time and reproduction rate were estimated for each of the experimental strains on a less favourable diet consisting of cracked wheat + cracked sorghum $(\mathrm{CW}+\mathrm{CS})$ in $1: 1$ ratio along with $5 \%$ yeast at $30{ }^{\circ} \mathrm{C}$ and $70 \% \mathrm{RH}$ (Jagadeesan et al. $2013 \mathrm{~b}$ ). The focus of this experiment was to examine possible impacts of resistance genes on the biological traits of insect strains grown under more challenging conditions. All the above experiments were replicated twice (experimental replicates) with three technical (sub) replicates each time.

\section{Data analysis}

The mortality responses of all the tested strains were subjected to probit analysis under generalised linear regression models (GenStat version 11, 2008) with/without $\log _{10}$ transformation, and the best fit was chosen based on key statistical parameters, slope, heterogeneity and deviance ratio. The $\mathrm{LC}_{50}$ and $\mathrm{LC}_{99.9}$ values were estimated from the best fitted probit regression curve for all the tested strains.

Data on the cumulative number of adults emerged from all the tested strains over a period of time were first converted to cumulative proportions and then fitted to a 3 -parameter logistic equation $Y=c /\left\{1+\exp \left(-b^{*}[X-m]\right)\right\}$, using a regression analysis with $X$ as days, which predicted the days to $50 \%$ emergence $(m)$, cumulative emergence $(Y)$, maximum emergence of progeny $(c)$ and the shape parameter (b) (Daglish et al. 2020). If a significant difference was found among the curves, they were refitted in a pairwise manner to determine whether there was significant difference between the individual pairs.
Total numbers of $F_{1}$ progeny emerged and weight of 100 insects in $\mathrm{mg}$ were analysed using an analysis of variance (ANOVA) (GenStat Version 11, 2008) after logarithmic $(N+1)$ transformation with strain as the treatment effect and experimental replicate as a block effect. If the treatment (strain) had significant effect, then a pairwise testing was performed using Fisher's protected least significant difference (LSD).

\section{Results}

\section{Phosphine bioassays}

Probit mortality response data of all the strains and $F_{24}$ with their $\mathrm{LC}_{50}$ and $\mathrm{LC}_{99.9}$ values reflected the phenotype of the strain (Table 1 and Fig. 2), and their higher mean deviance ratios $(P<0.001)$ and lower heterogeneity values (4.14-6.37) indicated the excellent fit into probit models. Logarithmic transformation of concentration as the explanatory variable resulted a better fit for the results of Ref-S and $F_{24}$, but not for the results of the Ref-R and Intro-R. The dose mortality response curve of Intro- $\mathrm{R}$ was parallel to Ref-R with a comparable $\mathrm{LC}_{50}\left(2.9 \mathrm{vs} 4.2 \mathrm{mg} \mathrm{L}^{-1}\right)$ and $\mathrm{LC}_{99.9}$ (6.0 vs $7.3 \mathrm{mg} \mathrm{L}^{-1}$ ). The Ref-R and Intro-R strains were $857 \times$ and $592 \times$ more resistant than the Ref-S, respectively. The $F_{24}$ segregating population, which likely had multiple genotypes, was found to be only $2.5 \times$ more resistant than the Ref-S strain at the $\mathrm{LC}_{50}$ level. However, the $\mathrm{LC}_{99.9}$ of the $F_{24}$ population was $37.8 \times$ that of the Ref-S strain confirming the presence of more tolerant genotypes.

\section{Phosphine resistance gene (cf_rph2) screening}

Sequence analysis of four randomly chosen individuals each from Ref-S, Ref-R and intro-R showed that all the four insects from Ref-R and Intro-R were homozygous for the $\mathrm{L} 73 \mathrm{~N}$ variant, while the individuals from Ref-S

Table 1 Probit mortality response of different strains of Cryptolestes ferrugineus to phosphine for $72 \mathrm{~h}$ at $25^{\circ} \mathrm{C}$ and $70 \% \mathrm{RH}$

\begin{tabular}{lllllllll}
\hline Strain & $n^{\mathrm{c}}$ & Slope $\pm \mathrm{SE}$ & $\mathrm{LC}_{50}(\mathrm{mg} / \mathrm{L})\left(95 \% \mathrm{FL}^{\mathrm{d}}\right)$ & $\mathrm{LC}_{99.9}(\mathrm{mg} / \mathrm{L})$ & $\begin{array}{l}\text { Resistance } \\
\text { Ratio at } \mathrm{LC}_{50}\end{array}$ & $\begin{array}{l}\text { Heterogene- } \\
\text { ity factor }\end{array}$ & $\begin{array}{l}\mathrm{df} \\
\text { Mean deviance ratio }\end{array}$ \\
\hline Ref-S $^{\mathrm{a}}$ & 3015 & $4.59 \pm 0.47$ & $0.005(0.0044-0.0054)$ & 0.023 & - & 5.98 & 8 & $262.7(P<0.001)$ \\
Ref-R $^{\mathrm{b}}$ & 4207 & $0.99 \pm 0.07$ & $4.2(4.1-4.4)$ & 7.3 & $857 \times$ & 6.37 & 10 & $527.6(P<0.001)$ \\
Intro-R $^{\mathrm{b}}$ & 4182 & $0.97 \pm 0.06$ & $2.9(2.7-3.0)$ & 6.0 & $592 \times$ & 4.14 & 10 & $686.3(P<0.001)$ \\
$\mathrm{F}_{24}{ }^{\mathrm{a}}$ & 3937 & $1.65 \pm 0.16$ & $0.012(0.008-0.015)$ & 0.87 & $2.45 \times$ & 4.72 & 11 & $225.5(P<0.001)$ \\
\hline
\end{tabular}

${ }^{\text {a }}$ Probit model used log concentration as explanatory variable

${ }^{\mathrm{b}}$ Probit model used concentration as explanatory variable

${ }^{\mathrm{c}}$ Total number of insects used in the bioassay

${ }^{\mathrm{d}}$ Fiducial limits 


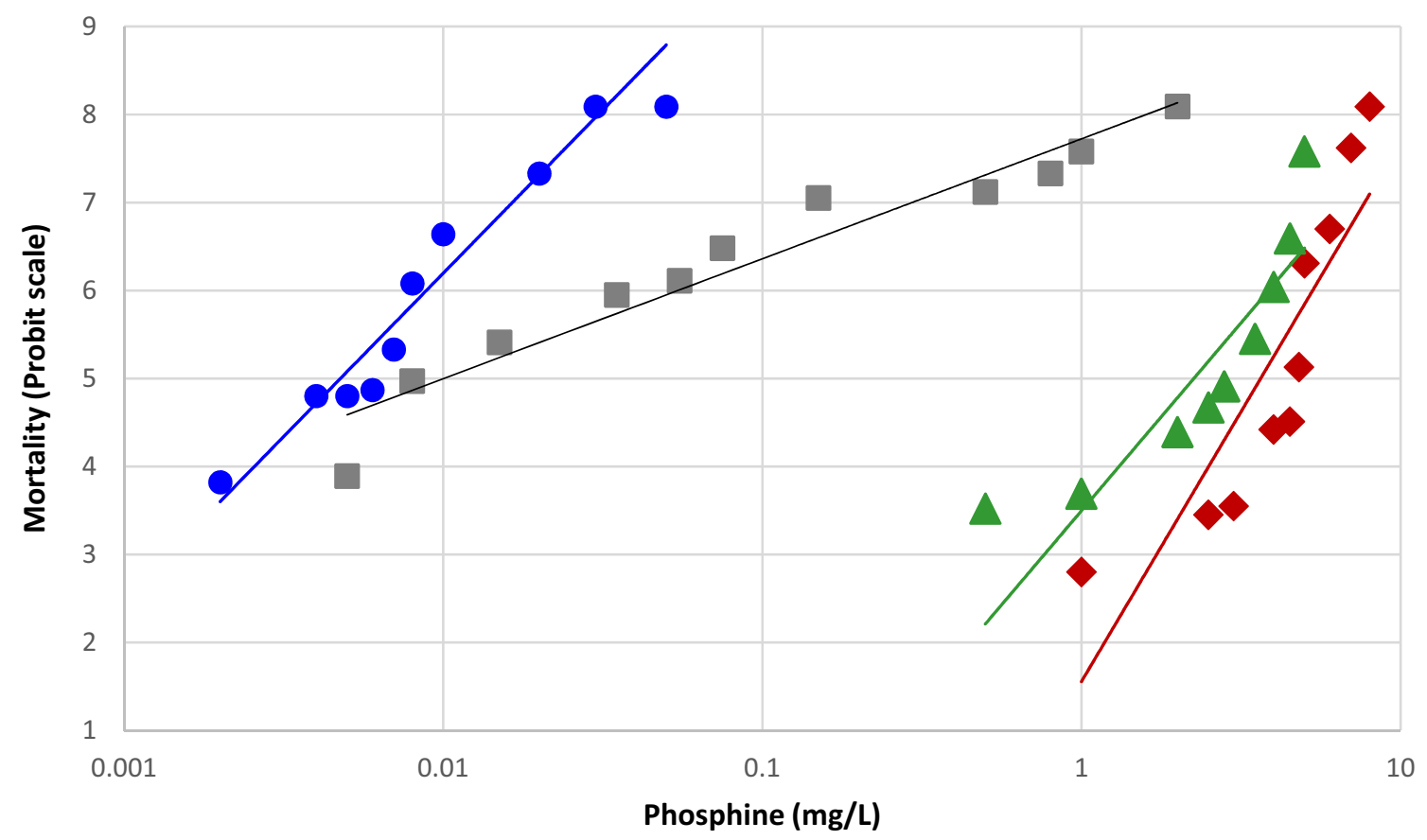

$\diamond$ Ref-R ORef-S $\Delta$ Intro-R $\quad$ F24

Fig. 2 Probit dose mortality response of different strains of Cryptolestes ferrugineus to phosphine for $72 \mathrm{~h}$ at $25{ }^{\circ} \mathrm{C}$ and $70 \% \mathrm{RH}$. Values in $X$ axis represent logarithmic concentration of phosphine in $\mathrm{mg} / \mathrm{L}$;

did not show this variant. This confirmed the successful introgression of the cf_rph2 allele in Intro-R.

\section{Developmental time}

\section{Optimal diet-rolled oats}

The data on cumulative emergence of progeny from tested strains and $F_{25}$ in the optimal diet of rolled oats fitted well with the 3-parameter logistic regression equation. At $30{ }^{\circ} \mathrm{C}$, the time to $50 \%$ emergence ranged from 24.6-27.1 days, with Intro-R and Ref-R showing 3.6 and $10.1 \%$ longer developmental time than Ref-S, respectively (Table 2). The pairwise analysis between Ref-S and Intro$\mathrm{R}$, with an aim to further evaluate the exclusive effect of resistance genes in isogenic background, revealed no significant difference in developmental time $(P=0.123)$ (Table 3$)$. In addition, the same comparative analysis between Ref-S and the $F_{25}(P=0.075)$ showed no significant differences. However, as anticipated, the curve for Ref-R was significantly different from both the Ref-S $(P<0.001)$ and $F_{25}(P<0.001)$ (Table 3$)$. values in $\mathrm{Y}$ axis are in probit scale in which $3.72,5.0$ and 8.09 correspond to 10,50 and $99.99 \%$ mortality

\section{Suboptimal diet-cracked wheat + cracked sorghum (CW+CS)}

Analysis of data obtained for Ref-S strain revealed significant variations in numbers of $F_{1}$ progeny produced between the two experimental replicates $(P<0.001)$. Therefore, data from each experimental replicate were analysed separately. Statistical outputs from first experimental replicate were skewed for significance in establishing 3-parameter logistic curves for all the strains and $F_{25}$ (Table 3), allowing us not to make any strong conclusions from this replicate. In experimental replicate 2, although the 3-parameter logistic curve analysis showed significant difference among the tested strains and $F_{25}$, the pairwise comparative analysis confirmed the absence of significant difference between the Ref-S and Intro-R strains $(P=0.065)$ and also between the Ref-S and Ref-R strains $(P=0.912)$. Interestingly, population of $F_{25}$ had a significantly shorter developmental time $(P<0.001)$ than the Ref-S. Extending this pairwise comparative analysis to experimental replicate 1 , confirmed the results of experimental replicate 2 (except for the Ref $S$ ), in showing shorter developmental time in $F_{25}(P<0.001)$ than the Ref-R and Intro-R (Table 3). 
Table 2 Regression analysis of cumulative $F_{1}$ adult emergence (proportions) from different strains of Cryptolestes ferrugineus at various experimental conditions

\begin{tabular}{lllllll}
\hline Experimental conditions & Strain & \multicolumn{2}{l}{ Parameters $( \pm$ SE) of logistic equation } & \multirow{2}{*}{$R^{2}$} & Significance \\
\cline { 3 - 5 } & & $m$ & $b$ & & \\
\hline Rolled Oats $30{ }^{\circ} \mathrm{C}$ & Ref-S & $24.6 \pm 0.828$ & $0.378 \pm 0.059$ & $0.937 \pm 0.124$ & 0.985 & $<0.001$ \\
& Ref-R & $27.1 \pm 0.316$ & $0.423 \pm 0.044$ & $0.925 \pm 0.049$ & & \\
& Intro-R & $25.5 \pm 0.605$ & $0.349 \pm 0.043$ & $1.07 \pm 0.098$ & & \\
EW +CS 30 ${ }^{\circ} \mathrm{C}$ & $F_{25}$ & $24.7 \pm 0.623$ & $0.460 \pm 0.072$ & $0.876 \pm 0.097$ & & \\
Experiment 1 & Ref-S & $29.6 \pm 0.221$ & $0.624 \pm 0.063$ & $1.01 \pm 0.031$ & 0.981 & $<0.001$ \\
& Ref-R & $42.4 \pm 0.738$ & $0.214 \pm 0.027$ & $1.19 \pm 0.094$ & & \\
CW +CS 30 ${ }^{\circ} \mathrm{C}$ & Intro-R & $40.8 \pm 0.383$ & $0.303 \pm 0.032$ & $1.06 \pm 0.048$ & & \\
Experiment 2 & $F_{25}$ & $36.4 \pm 0.270$ & $0.394 \pm 0.036$ & $1.02 \pm 0.030$ & & \\
& Ref-S & $40.7 \pm 0.439$ & $0.303 \pm 0.041$ & $0.984 \pm 0.049$ & 0.969 & $<0.001$ \\
& Ref-R & $40.9 \pm 0.437$ & $0.299 \pm 0.040$ & $1.00 \pm 0.049$ & & \\
Rolled Oats 22 ${ }^{\circ} \mathrm{C}$ & Intro-R & $38.9 \pm 0.380$ & $0.356 \pm 0.045$ & $0.992 \pm 0.043$ & & \\
& $F_{25}$ & $37.2 \pm 0.339$ & $0.446 \pm 0.055$ & $0.965 \pm 0.039$ & & \\
& Ref-S & $67.7 \pm 0.902$ & $0.348 \pm 0.089$ & $0.978 \pm 0.089$ & 0.867 & 0.985 \\
& Ref-R & $67.9 \pm 1.00$ & $0.324 \pm 0.085$ & $0.976 \pm 0.097$ & & \\
& Intro-R & $68.6 \pm 1.30$ & $0.275 \pm 0.072$ & $1.02 \pm 0.124$ & & \\
\hline & $F_{25}$ & $67.7 \pm 1.20$ & $0.278 \pm 0.071$ & $1.00 \pm 0.111$ & & \\
\hline
\end{tabular}

Rolled oats $30{ }^{\circ} \mathrm{C}$ represent insects grown in a regular diet of rolled oats and yeast (20:1) at optimal temperature of $30^{\circ} \mathrm{C}$

$\mathrm{CW}+\mathrm{CS} 30^{\circ} \mathrm{C}$ represents insects grown in a less favourable diet of cracked wheat and cracked sorghum in $1: 1$ ratio along with $5 \%$ yeast at $30^{\circ} \mathrm{C}$

Rolled oats $22^{\circ} \mathrm{C}$ represent insects grown in a regular diet of rolled oats and yeast (20:1) at suboptimal low temperature of $22{ }^{\circ} \mathrm{C}$

$m$ is days to $50 \%$ emergence of adults; $b$ is curve steepness and $c$ is maximum emergence derived from cumulative proportions emerged

Table 3 Comparison of regression analysis of cumulative $F_{1}$ adult emergence (proportions) between individual pairs of Cryptolestes ferrugineus when the regression among group of strains was significant

\begin{tabular}{lllllll}
\hline Experimental conditions & $P$ value & & \\
\cline { 2 - 6 } & Ref-S and Intro-R & Ref-S and $F_{25}$ & Ref-S and Ref-R & Ref-R and Intro-R & Ref-R and $F_{25}$ & Intro-R and $F_{25}$ \\
\hline Rolled oats $30^{\circ} \mathrm{C}$ & 0.123 & 0.075 & $<0.001^{* * *}$ & 0.073 & $<0.001^{* * *}$ & $0.002^{* *}$ \\
$\mathrm{CW}+\mathrm{CS} 30^{\circ} \mathrm{C}$ Experiment 1 & $<0.001^{* * *}$ & $<0.001^{* * *}$ & $<0.001^{* * *}$ & $0.027^{*}$ & $<0.001^{* * *}$ & $<0.001^{* * *}$ \\
$\mathrm{CW}+\mathrm{CS} 30^{\circ} \mathrm{C}$ Experiment 2 & 0.065 & $<0.001^{* * *}$ & 0.912 & $<0.001^{* * *}$ & $<0.001^{* * *}$ & $<0.001^{* * *}$ \\
\hline
\end{tabular}

Rolled oats $30^{\circ} \mathrm{C}$ represent insects grown in a regular diet of rolled oats and yeast (20:1) at optimal temperature of $30{ }^{\circ} \mathrm{C}$

$\mathrm{CW}+\mathrm{CS} 30{ }^{\circ} \mathrm{C}$ represents insects grown in a less favourable diet of cracked wheat and cracked sorghum in 1:1 ratio along with $5 \%$ yeast at $30{ }^{\circ} \mathrm{C}$

\section{Suboptimal low temperature- $22{ }^{\circ} \mathrm{C}$}

Challenging the experimental strains at the suboptimal temperature $\left(22^{\circ} \mathrm{C}\right)$ resulted in longer developmental time in all the strains (67.7-68.6 days) with no significant difference among them $(P=0.985)$ (Table 2$)$.

\section{Reproduction and body weight}

On the optimal diet of rolled oats at $30{ }^{\circ} \mathrm{C}$, there was no significant difference between the total numbers of $F_{1}$ progeny emerged in all strains $\left(F_{3,7}=1.49, P=0.376\right)$. However, the effect of strain on individual beetle body weight was significant only between the Ref-S and Ref-R $\left(F_{3,7}=13.9, P=0.029\right)$ with Ref-R having the least body weight (Table 4$)$. On the suboptimal diet $(\mathrm{CW}+\mathrm{CS})$, no significant effect of strain was observed with either $F_{1}$ progeny $\left(F_{3,7}=7.37, P=0.068\right)$ or beetle weight $\left(F_{3,7}=0.73\right.$, $P=0.599$ ). At the suboptimal low temperature of $22{ }^{\circ} \mathrm{C}$, however, while the effect of strain on total numbers of $F_{1}$ progeny was not significant $\left(F_{3,7}=7.25, P=0.069\right)$, there was significant difference in beetle body weight $\left(F_{3,7}=502\right.$, 
Table 4 Reproduction and test weight of $F_{1}($ mean $\pm \mathrm{SD}, \mathrm{n}=2)$ progeny of different strains of Cryptolestes ferrugineus at various experimental conditions

\begin{tabular}{llll}
\hline Experimental conditions & Strain & $\begin{array}{l}\text { Total } \mathrm{F}_{1} \text { adults }(\log [N+1] \\
\text { transformed })\end{array}$ & $\begin{array}{l}\text { Weight of } 100 \mathrm{~F}_{1} \text { adults } \\
(\mathrm{mg})(\log [N+1] \text { trans- } \\
\text { formed })\end{array}$ \\
\hline Rolled Oats $30{ }^{\circ} \mathrm{C}$ & Ref-S & $6.97 \pm 0.16^{\mathrm{a}}$ & $3.71 \pm 0.09^{\mathrm{a}}$ \\
& Ref-R & $6.88 \pm 0.20^{\mathrm{a}}$ & $3.50 \pm 0.01^{\mathrm{b}}$ \\
& Intro-R & $7.10 \pm 0.04^{\mathrm{a}}$ & $3.64 \pm 0.06^{\mathrm{a}}$ \\
$\mathrm{CW}+\mathrm{CS} 30{ }^{\circ} \mathrm{C}$ & $F_{25}$ & $6.97 \pm 0.08^{\mathrm{a}}$ & $3.67 \pm 0.08^{\mathrm{a}}$ \\
& Ref-S & $5.61 \pm 0.27^{\mathrm{a}}$ & $3.07 \pm 0.02^{\mathrm{a}}$ \\
& Ref-R & $5.76 \pm 0.03^{\mathrm{a}}$ & $3.09 \pm 0.12^{\mathrm{a}}$ \\
& Intro-R & $5.99 \pm 0.07 \mathrm{a}$ & $3.09 \pm 0.12^{\mathrm{a}}$ \\
& $F_{25}$ & $6.07 \pm 0.19^{\mathrm{a}}$ & $3.17 \pm 0.01^{\mathrm{a}}$ \\
& Ref-S & $5.03 \pm 1.54^{\mathrm{a}}$ & $3.57 \pm 0.003^{\mathrm{b}}$ \\
& Ref-R & $4.23 \pm 1.01^{\mathrm{a}}$ & $3.53 \pm 0.001^{\mathrm{c}}$ \\
& Intro-R & $4.33 \pm 1.21^{\mathrm{a}}$ & $3.58 \pm 0.006^{\mathrm{b}}$ \\
& $F_{25}$ & $5.52 \pm 0.79^{\mathrm{a}}$ & $3.64 \pm 0.001^{\mathrm{a}}$ \\
\hline
\end{tabular}

Rolled oats $30{ }^{\circ} \mathrm{C}$ represent insects grown in a regular diet of rolled oats and yeast (20:1) at optimal temperature of $30^{\circ} \mathrm{C}$

$\mathrm{CW}+\mathrm{CS} 30^{\circ} \mathrm{C}$ represents insects grown in a less favourable diet of cracked wheat and cracked sorghum in $1: 1$ ratio along with $5 \%$ yeast at $30^{\circ} \mathrm{C}$

Rolled oats $22{ }^{\circ} \mathrm{C}$ represent insects grown in a regular diet of rolled oats and yeast (20:1) at suboptimal low temperature of $22^{\circ} \mathrm{C}$

Within experimental conditions and column, means followed by same letters are not significantly different $(P<0.05)$
$P<0.002$ ) recorded among different strains. However, the Ref-S and Intro-R had similar body weight and the $F_{25}$ being the heaviest (Table 4).

\section{Discussion}

The hypothesis that phosphine resistant insects may possibly have a fitness cost was investigated in $C$. ferrugineus with the control over background genetic effects in optimal and suboptimal conditions. We have used the gene introgression approach to produce a near isogenic resistant strain, which shared the similar genetic background with the susceptible strain except for the phosphine resistance genes. Moreover, inclusion of a segregating population $\left(F_{25}\right)$ generated from the crossing of the Ref-S and Ref-R strains allowed us testing a population having multiple genotypes. This population potentially had nine genotypes and therefore may reflect field populations more closely than a homozygous resistant strain. In addition, the gene recombinations over 24 generations in this segregating population would have provided partial control for background genetic factors (Roush and McKenzie 1987).

Both the phenotypic and genetic characterisation for phosphine resistance confirmed that the established Intro- $R$ was strongly resistant with a resistance factor of $592 \times$, comparable to the Ref-R with a resistance factor of $857 \times$ that was highly homozygous for the cf_rph2 allele, L73N. These results explicitly validate our approach in establishing nearisogenic phosphine resistant insect strain, especially for investigating fitness effects. Although many studies had adopted the gene introgression approach to investigate the fitness cost (Arnaud et al. 2005; Horikoshi et al. 2016), very few of them in fact validated the success of this approach phenotypically (White and Bell 1990) or genotypically (Berticat et al. 2002). Recently, Daglish et al. (2020) employed the gene introgression approach to produce a near-isogenic resistant strain of $T$. castaneum and confirmed the gene introgression through phenotypic and genotypic characterisation similar to what was used in the current study. In general, the success of introgression approach relies on the number of recurrent backcrosses $\left(F_{1}\right.$-BC), followed by phosphine selection on progeny $\left(F_{2}\right.$-BC). In the current study, the introgression of genes was achieved with only three rounds of repeated backcrossing. This is relatively shorter compared to the approaches adopted previously (Berticat et al. 2002; Arnaud et al. 2005; Horikoshi et al. 2016) suggesting that the current approach can be adopted in future to construct near-isogenic resistant strain for other stored product pests.

Although there were asynchronous emergence of adults among the strains with Ref-R emerging late, our statistical analysis proved that the Intro-R and the $F_{25}$ were not significantly different to the Ref-S in developmental time, numbers of $F_{1}$ emerged and body weight, confirming that the resistance genes of $C$. ferrugineus are unlikely to incur any fitness cost. Reproduction was assessed following 1 week of 
oviposition. Future research could examine the possibility that a fitness effect on reproduction is manifested over longer time-spans. Our results supported the importance of controlling for background genetic effects, without which the significant differences observed between the Ref-R and Ref-S in developmental time and body weight could have been interpreted that phosphine resistance genes are associated with fitness costs. Daglish et al. (2020) also observed significant difference in beetle weight between susceptible and resistant strains in T. castaneum, but not when the phosphine resistance trait was compared in an isogenic background, supporting the conclusion of this study. Similar results of insecticide susceptible strain having fitness advantage over the resistant strain but not with the near-isogenic resistant strain were reported in resistance to malathion in C. ferrugineus (White and Bell 1990) and Bt toxins (Cry1F) in the fall army worm, Spodoptera frugiperda (J.E. Smith) (Dangal and Huang 2015; Horikoshi et al. 2016). Taken these reports into consideration, it can be concluded that establishing a near-isogenic resistant insect strain is crucial in investigating fitness costs associated with an insecticide resistance trait. Several earlier studies reported significant fitness cost associated with phosphine resistance in T. castaneum, $R$. dominica and $O$. surinamensis (Pimentel et al. 2007; Sousa et al. 2009; Bajracharya et al. 2016) but without controlling for genetic background. Based on our present results, we believe that such results need to be viewed with caution and suggest that future investigations on fitness should include near-isogenic resistant lines.

While we conclude that phosphine resistant insects do not incur fitness costs in $C$. ferrugineus, in alignment with previous reports on three major stored product pest species, $R$. dominica, T. castaneum and S. oryzae (Schlipalius et al. 2008; Daglish et al. 2014, 2015, 2020), the observed decline in $r p h 2$ allele frequency in unselected segregating populations of T. castaneum (Jagadeesan et al. 2013a) and S. oryzae (Nguyen et al. 2016) needs further examination. Unfortunately, both these latter studies could not continue assessing the decline in allele frequency beyond the $F_{20}$ filial generations, and did not correlate the observed change in allele frequency in terms of developmental or reproductive traits. Although the authors correlated strength of resistance phenotypically, we suggest that future studies on fitness should be designed to assess both the changes in frequency of resistant alleles and its effect on developmental and reproductive trait. Such studies will reveal the existence of insignificant fitness cost that can be cumulative over generations.

With the perspective that fitness costs are likely to be more evident in stressed environments (Raymond et al. 2005,2011 ), we challenged the insect strains with a suboptimal diet and suboptimal low temperature. Neither rearing $C$. ferrugineus on a suboptimal diet $(\mathrm{CW}+\mathrm{CS})$ nor exposing them to a suboptimal low temperature $\left(22^{\circ} \mathrm{C}\right)$ revealed specific fitness differences in terms of development time and progeny production between the strains, especially between Intro-R and Ref-S. Despite the fact that while both of the suboptimal conditions substantially delayed the developmental time and fecundity of all experimental insect strains, there was no fitness cost linked to the phosphine resistance genes. These results emphasise that the conclusion of absence of fitness cost associated with phosphine resistance remains the same under suboptimal conditions, as observed in previous studies that have stressed phosphine resistant insects in a less favourable diet (Daglish et al. 2020) or through starvation (Kaur et al. 2013). On the contrary, specific costs associated with insecticide resistance only in stressed environments such as unfavourable host plant (Raymond et al. 2011; Silva et al. 2012) or at cold temperatures (Carrière et al. 2001; $\mathrm{Li}$ et al. 2007), have been reported in various crop pests. Hence, we conclude that the pleiotropic effects of fitness costs associated with resistance genes under challenging environments could vary widely among insect species.

Mechanism of resistance to phosphine involves the synergistic interaction of two genes cf_rph1 and cf_rph2 that are directly involved in energy metabolism (Schlipalius et al. 2012, 2018). Therefore, it is obvious to assume that resistant insects divert their energy resources to survive phosphine fumigations, causing at least minimal metabolic deficits. However, our current findings for $C$. ferrugineus suggest that resistant insects of this species are unlikely to have any deleterious effect on development and reproduction. Thus, we propose that further research be directed towards exploring the specific biochemical or metabolic responses related to fitness in resistant insects in both the presence and absence of phosphine exposure. The other possibility is that continuous selection for phosphine resistance could have resulted in selection for some other genes, referred as fitness modifiers (McKenzie et al. 1982), which might have possibly compensated the fitness costs in case of $C$. ferrugineus. One of the recommended resistance management tactics is to suspend phosphine usage for extended periods, which would allow the susceptible alleles to increase in the population (Tabashnik 1989). Our results, however, suggest that this tactic may not be very effective in case of $C$. ferrugineus, as phosphine resistance frequency would be stable in field populations. We propose that using a break fumigant, with completely different mode of action, such as sulfuryl fluoride, can be recommended as this may reduce selection pressure to phosphine in insects and possibly eradicate fully resistant genotypes.

\section{Author contributions}

VS, RJ and GD conceived and designed research, VS and RJ performed analysis and wrote manuscript, GD, MN and 
PE revised the manuscript significantly. All authors read and approved the manuscript.

Acknowledgements With regard to the development of the introgressed resistant strain, the authors gratefully acknowledge the support of plant biosecurity cooperative research centre (Project PBCRC3039), established and supported under the Australian Government's Cooperative Research Centres program (http://www.crcplantbiosecurity. com.au). With regard to the investigation of potential fitness costs, the authors gratefully acknowledge the support of the Australian Government's Australia-India strategic research fund (AISRF 48516), Department of Industry and Science (DIS), Canberra. The authors would also like to acknowledge Kerri Chandra for assisting with statistical analysis, David Schlipalius for designing primers for molecular screening and Lui Lawrence Rangger for technical assistance.

Data Availability The datasets generated during the current study are available from the corresponding author on reasonable request.

\section{Compliance with Ethical Standards}

Conflicts of interest All authors declare that they have no conflict of interest.

Ethical approval This article does not contain any studies with human participants or animals performed by any of the authors.

Consent to participate Not applicable as it involves no human research participants.

Consent for publication Not applicable as it involves no human research participants.

\section{References}

Afful E, Elliott B, Nayak MK, Phillips TW (2018) Phosphine resistance in North American field populations of the lesser grain borer, Rhyzopertha dominica (F.) (Coleoptera: Bostrichidae). J Econ Entomol 111:463-469

Agrafioti P, Athanassiou CG, Nayak MK (2019) Detection of phosphine resistance in major stored-product insects in Greece and evaluation of a field resistance test kit. J Stored Prod Res 82:40-47

Alyokhin AV, Ferro DN (1999) Relative fitness of Colorado potato beetle (Coleoptera: Chrysomelidae) resistant and susceptible to the Bacillus thuringiensis Cry3A toxin. J Econ Entomol 92:510-515

Arnaud L, Haubruge E, Gage MJG (2005) The malathion specific resistance gene confers a sperm competition advantage in tribolium castaneum. Funct Ecol 19:1032-1039

Ashby KR (1961) The population dynamics of Cryptolestes ferrugineus (Stephens) in flour and on Manitoba wheat. Bull Entomol Res 52:363-379

Bajracharya NS, Opit GP, Talley J, Gautam SG, Payton ME (2016) Assessment of fitness effects associated with phosphine resistance in Rhyzopertha dominica (F.) (Coleoptera: Bostrichidae) and Tribolium castaneum (Herbst) (Coleoptera: Tenebrionidae). African Entomology 24:39-49

Benhalima H, Choudhry MQ, Mills KA, Price NR (2004) Phosphine resistance in stored-product insects collected from various grain facilities in Morocco. J Stored Prod Res 40:241-249
Berticat C, Boquien G, Raymond M, Chevillon C (2002) Insecticide resistance genes induce a mating competition cost in Culex pipiens mosquitoes. Genet Res Camb 79:41-47

Carrière Y, Ellers-Kirk C, Patin AL, Sims MA, Meyer S, Liu YB, Dennehy TJ, Tabashnik BE (2001) Overwintering costs associated with resistance to transgenic cotton in the pink bollworm. J Econ Entomol 94:935-941

Chaudhry MQ, Price NR (1992) Comparison of the oxidant damage induced by phosphine and the uptake and tracheal exchange of $\mathrm{P}-32$ radiolabeled phosphine in the susceptible and resistant strains of Rhyzopertha dominica (F) (Coleoptera, Bostrychidae). Pestic Biochem Physiol 42:167-179

Collins PJ, Falk MG, Nayak MK, Emery RN, Holloway JC (2017) Monitoring resistance to phosphine in the lesser grain borer, Rhyzopertha dominica, in Australia: A national analysis of trends, storage types and geography in relation to resistance detections. J Stored Prod Res 70:25-36

Daglish GJ, Nayak MK, Pavic H (2014) Phosphine resistance in Sitophilus oryzae (L.) from eastern Australia: inheritance, fitness and prevalence. J Stored Prod Res 59:237-244

Daglish GJ, Nayak MK, Pavic H, Smith LW (2015) Prevalence and potential fitness cost of weak phosphine resistance in Tribolium castaneum (Herbst) in eastern Australia. J Stored Prod Res 61:54-58

Daglish GJ, Jagadeesan R, Nayak MK, McCulloch GJ, Singarayan VT, Walter GH (2020) The gene introgression approach and the potential cost of genes that confer strong phosphine resistance in red flour beetle. J. Econ. Entomol, Tribolium castaneum (Herbst). https://doi.org/10.1093/jee/toaa033

Dangal V, Huang F (2015) Fitness costs of Cry1F resistance in two populations of fall armyworm, Spodoptera frugiperda (J.E Smith), collected from Puerto Rico and Florida. J Invertebr Pathol 127:81-86

Fields PG (1992) The control of stored-product insects and mites with extreme temperatures. J Stored Prod Res 28:89-118

Gassmann AJ, Carrière Y, Tabashnik BE (2009) Fitness costs of insect resistance to Bacillus thuringiensis. Annu Rev Entomol 54:147-163

GenStat Version 11 (2008) GenStat for windows, release 11.1. VSN International Ltd., Oxford

Holloway JC, Falk MG, Emery RN, Collins PJ, Nayak MK (2016) Resistance to phosphine in Sitophilus oryzae in Australia: A national analysis of trends and frequencies over time and geographic spread. J Stored Prod Res 69:129-137

Horikoshi RJ, Bernardi O, Bernardi D, Okuma DM, Farias JR, Miraldo LL, Amaral FS, Omoto C (2016) Near-isogenic cry1f-resistant strain of Spodoptera frugiperda (Lepidoptera: Noctuidae) to investigate fitness cost associated with resistance in Brazil. J Econ Entomol 109:854-859

Jagadeesan R, Nayak MK (2017) Phosphine resistance does not confer cross-resistance to sulfuryl fluoride in four major stored grain insect pests. Pest Manag Sci 73:1391-1401

Jagadeesan R, Fotheringham A, Ebert PR, Schlipalius DI (2013a) Rapid genome wide mapping of phosphine resistance loci by a simple regional averaging analysis in the red flour beetle, Tribolium castaneum. BMC Genomics 14:1471-2164

Jagadeesan R, Nayak MK, Dawson K, Byrne V, Collins PJ (2013b) Dietary media for mass rearing of rusty grain beetle, Cryptolestes ferrugineus (Stephens) and flat grain beetle, Cryptolestes pusillus (Schonherr) (Coleoptera). J Stored Prod Res 55:68-72

Jagadeesan R, Collins PJ, Nayak MK, Schlipalius DI, Ebert PR (2016) Genetic characterization of field-evolved resistance to phosphine in the rusty grain beetle, Cryptolestes ferrugineus (Laemophloeidae: Coleoptera). Pest Biochem Physiol 127:67-75

Jagadeesan R, Schlipalius DI, Singarayan VT, Nath NS, Nayak MK, Ebert PR (2020) Unique genetic variants in dihydrolipoamide 
dehydrogenase (dld) gene confer strong resistance to phosphine in the rusty grain beetle, Cryptolestes ferrugineus (Stephens). Pestic Biochem Physiol 104717

Kaur R, Nayak MK (2015) Developing effective fumigation protocols to manage strongly phosphine-resistant Cryptolestes ferrugineus (Stephens) (Coleoptera: Laemophloeidae). Pest Manag Sci 71:1297-1302

Kaur R, Ebert PE, Walter HH, Swain AJ, Schlipalius DI (2013) Do phosphine resistance genes influence movement and dispersal under starvation? J Econ Entomol 100:2259-2266

Kaur R, Subbarayalu M, Jagadeesan R, Daglish GJ, Nayak MK et al (2015) Phosphine resistance in India is characterised by a dihydrolipamide dehydrogenase variant that is otherwise unobserved in eukaryotes. Heredity 115:188-194

Koçak E, Schlipalius D, Kaur R, Tuck A, Ebert P et al (2015) Determining phosphine resistance in rust red flour beetle, Tribolium castaneum (Herbst.) (Coleoptera : Tenebrionidae) populations from Turkey. Türk Entomol Derg 39:129-136

Konemann CE, Hubhachen Z, Opit GP, Gautam SG, Bajracharya NS (2017) Phosphine resistance in Cryptolestes ferrugineus (Coleoptera: Laemophloeidae) collected from grain storage facilities in Oklahoma. USA J Econ Entomol 110:1377-1383

Li ZM, Liu SS, Liu YQ, Ye GY (2007) Temperature-related fitness costs of resistance to spinosad in the diamondback moth, Plutella xylostella (Lepidoptera: Plutelidae). Bull Entomol Res 97:627-635

Lorini I, Collins PJ, Daglish GJ, Nayak MK, Pavic H (2007) Detection and characterisation of strong resistance to phosphine in Brazilian Rhyzopertha dominica (F.) (Coleoptera: Bostrychidae). Pest Manag Sci 63:358-364

Malekpour R, Rafter MA, Daglish GJ, Walter GH (2018) The movement abilities and resource location behaviour of Tribolium castaneum: phosphine resistance and its genetic influences. J Pest Sci 91:739-749

McKenzie JA (1996) Ecological and evolutionary aspects of insecticide resistance. R.G Landes Company, Austin

McKenzie JA, Whitten MJ, Adena MA (1982) The effect of genetic background on the fitness of diazinon resistance genotypes of the Australian sheep blowfly, Lucilia cuprina. Heredity 49:1-9

Montero-Pau J, Gomez A (2008) Application of an inexpensive and high-throughput genomic DNA extraction method for the molecular ecology of zooplanktonic diapausing eggs. Limnol Oceanogr Methods 6:218-222

Nayak MK, Holloway JC, Emery RN, Pavic H, Bartlet J, Collins PJ (2013) Strong resistance to phosphine in the rusty grain beetle, Cryptolestes ferrugineus (Stephens) (Coleoptera: Laemophloeidae): its characterisation, a rapid assay for diagnosis and its distribution in Australia. Pest Manag Sci 69:48-53

Nayak MK, Falk MG, Emery RN, Collins PJ, Holloway JC (2017) An analysis of trends, frequencies and factors influencing the development of resistance to phosphine in the red flour beetle Tribolium castaneum (Herbst) in Australia. J Stored Prod Res 72:35-48

Nayak MK, Daglish GJ, Phillips TW, Ebert PR (2020) Resistance to the fumigant phosphine and its management in insect pests of stored products: A global perspective. Ann Rev Entomol 65:333-350

Nguyen T, Collins PJ, DuongTM SD, Ebert PR (2016) Genetic conservation of phosphine resistance in the rice weevil, Sitophilus oryzae (L). J Hered 107:228-237

Oliveira EE, Guedes RNC, Totola MR, De Marco P (2007) Competition between insecticide-susceptible and-resistant populations of the maize weevil, Sitophilus zeamais. Chemosphere 69:17-24

Pimentel MAG, D'A Faroni LR, Totola MR, Guedes RNC, (2007) Phosphine resistance, respiration rate and fitness consequences in stored-product insects. Pest Manag Sci 63:876-881
Raymond B, Sayyed AH, Wright DJ (2005) Genes and environment interact to determine the fitness costs of resistance to Bacillus thuringiensis. Proc R Soc B 272:1519-1524

Raymond B, Wright DJ, Bonsall MB (2011) Effects of host plant and genetic background on the fitness costs of resistance to Bacillus thuringiensis. Heredity 106:281-288

Rilett RO (1949) The biology of Laemophloeus ferrugineus (Steph.). Can J Res 27:112-148

Roush RT, McKenzie JA (1987) Ecological genetics of insecticide and acaricide resistance. Annu Rev Entomol 32:361-380

Sakka MK, Romano D, Stefanini C, Canale A, Benelli G, Athanassiou C (2020) Mobility parameters of Tribolium castaneum and Rhyzopertha dominica populations with different susceptibility to phosphine. J Stored Prod Res 87:101593

Schlipalius DI, Cheng Q, Reilly PE, Collins PJ, Ebert PR (2002) Genetic linkage analysis of the lesser grain borer Rhyzopertha dominica identifies two loci that confer high-level resistance to the fumigant phosphine. Genetics 161:773-782

Schlipalius DI, Chen W, Collins PJ, Nguyen T, Reilly PEB, Ebert PR (2008) Gene interactions constrain the course of evolution of phosphine resistance in the lesser grain borer, Rhyzopertha dominica. Heredity 100:506-516

Schlipalius DI, Valmas N, Tuck AG, Jagadeesan R, Ma L, Kaur R, Goldinger A, Anderson C, Kuang J, Zuryn S et al (2012) A core metabolic enzyme mediates resistance to phosphine gas. Science 338:807-810

Schlipalius DI, Tuck A, Jagadeesan R, Nguyen T, Kaur R, Subramanian $\mathrm{S}$ et al (2018) Variant linkage analysis using de Novo transcriptome sequencing identifies a conserved phosphine resistance gene in insects. Genetics 209:281-290

Silva AX, Bacigalupe LD, Luna-Rudloff M, Figueroa C (2012) Insecticide resistance mechanisms in the Green Peach Aphid Myzus persicae (Hemiptera: Aphididae) II: Costs and benefits. PLoS ONE 7(6):e36810

Sousa AH, Faroni LR, Pimentel MAG, Guedes RNC (2009) Developmental and population growth rates of phosphine-resistant and -susceptible populations of stored-product insect pests. J Stored Prod Res 45:241-246

Tabashnik BE (1989) Managing resistance with multiple pesticide tactics: theory, evidence and recommendations. J Econ Entomol 82:1263-1269

White NDG, Bell RJ (1990) Relative fitness of a malathion resistant strain of Cryptolestes ferrugineus (Coleoptera: Cucujidae) when development and oviposition occur in malathion-treated and untreated wheat kernels. J Stored Prod Res 26:23-37

White NDG, Collin DJ, Kawamoto H, Sinha RN (1995) Population growth of Cryptolestes ferrugineus and C. pusillus (Coleoptera: Cucujidae) alone, or in competition in stored wheat or maize at different temperatures. Bull Entomol Res 85:425-429

Zhu X, Yang Y, Wu Q, Wang S, Xie W, Guo Z, Kang S, Xia J, Zhang Y (2016) Lack of fitness costs and inheritance of resistance to Bacillus thuringiensis Cry1Ac toxin in a near-isogenic strain of Plutella xylostella (Lepidoptera: Plutellidae). Pest Manag Sci 72:289-297

Publisher's Note Springer Nature remains neutral with regard to jurisdictional claims in published maps and institutional affiliations. 